\title{
Decreased Cardiac Vagal Control in Drug-Naïve Patients with Posttraumatic Stress Disorder
}

\author{
Hsin-An Chang ${ }^{1}$, Chuan-Chia Chang ${ }^{1}$, Nian-Sheng Tzeng ${ }^{1,2}$, \\ Terry BJ Kuo ${ }^{3}$, Ru-Band $\mathrm{Lu}^{4}$ and San-Yuan Huang ${ }^{1 凶}$ \\ 1Department of Psychiatry, Tri-Service General Hospital, National Defense Medical Center, Taipei, Taiwan \\ ${ }^{2}$ Student Counseling Center, National Defense Medical Center, Taipei, Taiwan \\ ${ }^{3}$ Institute of Brain Science, National Yang-Ming University, Taipei, Taiwan \\ ${ }^{4}$ Institute of Behavioral Medicine and Department of Psychiatry, College of Medicine, National Cheng Kung University, Tainan, Taiwan
}

Objective Decreased cardiac vagal control (CVC) has been proposed in posttraumatic stress disorder (PTSD), but the results are mixed. Analyses with larger sample sizes and better methodology are needed.

Methods Thirty-two drug-naïve survivors with current PTSD, 32 survivors without PTSD and 192 matched controls were recruited for a case-control analysis. We used the PTSD checklist-civilian version (PCL-C) to assess posttraumatic symptoms severity. Cardiac autonomic function was evaluated by measuring heart rate variability (HRV) parameters. Frequency-domain indices of HRV were obtained. The obtained results were evaluated in association with personality traits assessed by the Tridimensional Personality Questionnaire (TPQ).

Results PTSD patients exhibited decreased LF-HRV and HF-HRV as compared to survivors without PTSD and to matched controls. The PTSD symptoms severity was associated with reduced mean RR intervals, Var-HRV, LF-HRV and HF-HRV. The harm avoidance score (which has been suggested to be associated with serotonergic activity) was negatively correlated with Var-HRV, LF-HRV and HF-HRV.

Conclusion These data suggest that PTSD is accompanied by decreased CVC, highlighting the importance of assessing HRV in PTSD patients. In view of the increased risk for cardiovascular diseases in these vulnerable individuals, one might consider the treatment to restore their autonomic function while reducing PTSD symptoms.

Psychiatry Investig 2013;10:121-130

Key Words Posttraumatic stress disorder, Heart rate variability, Cardiac autonomic function, Vagal control.

\section{INTRODUCTION}

Posttraumatic stress disorder (PTSD) is a debilitating anxiety disorder which causes significant work and social dysfunction. ${ }^{1}$ The three main dimensions of PTSD are re-experiencing the traumatic event, avoidance of stimuli associated with the event and numbing of emotions, and increased arousal. Due to the prominence of hyperarousal symptoms in PTSD, previous research has focused primarily on the sympathetic branch of the autonomic nervous system (ANS). Most studies

Received: September 12, 2012 Revised: November 25, 2012

Accepted: November 28, 2012 Available online: May 30, 2013

$\triangle$ Correspondence: San-Yuan Huang, MD, PhD

Professor and Attending Psychiatrist, Department of Psychiatry, Tri-Service General Hospital, No. 325, Cheng-Kung Road, Sec. 2, Nei-Hu District, Taipei, 114, Taiwan, ROC

Tel: +011-886-2-8792-7220, Fax: +011-886-2-8792-7221

E-mail: chang.ha@msa.hinet.net

(a) This is an Open Access article distributed under the terms of the Creative Commons Attribution Non-Commercial License (http://creativecommons.org/licenses/by$\mathrm{nc} / 3.0$ ) which permits unrestricted non-commercial use, distribution, and reproduction in any medium, provided the original work is properly cited. consistently showed higher levels of resting heart rate and skin conductance $^{2}$ and an elevated phasic activation of the sympathetic nervous system in response to trauma-related cues ${ }^{3}$ in PTSD patients. However, Murburg et al. ${ }^{4}$ argued that baseline sympathetic activity was not increased in PTSD patients and that previous reports of increased resting sympathetic activity in this population might instead reflect sympathetic reactivity.

With regard of the parasympathetic nervous system (PNS), the other branch the ANS, it has been overlooked previously in the psychophysiologic investigation of PTSD. The PNS, and especially the vagal regulation of heart rate, is involved in stress reactions and the long-term consequences of stress. ${ }^{5}$ These vagal fibers functionally slow heart rate and actively inhibit the sympathetic influences to the heart. ${ }^{5}$ Cardiac vagal control (CVC), essentially, reflects the degree to which there is tonic vagal influence on the heart ${ }^{6}$ and is indexed by estimates of respiratory sinus arrhythmia (RSA, a time domain measure of heart rate variability) or high frequency power of spectral an- 
alysis of heart rate variability (HF-HRV). Decreased HF-HRV, which causes reduced autonomic flexibility, is associated with increased cardiac mortality after myocardial infarction and the development of serious ventricular arrhythmias. In recent years, CVC has become a promising candidate for investigation and there is growing evidence highlighting its importance in PTSD. For example, survivors of road traffic accidents exhibiting cardiac vagal dysfunction as assessed from heart rate time series, were reported more susceptible to developing PTSD. ${ }^{7}$ Similarly, fire services workers with PTSD showed a significantly lower cardiac parasympathetic nervous activity than controls at baseline. ${ }^{8}$ Accordingly, one might wonder the relevance of cardiac vagal tone to PTSD.

In studying the ANS, various techniques have been developed to detect the function of the sympathetic and parasympathetic systems. Frequency-domain analysis of HRV, with its standard procedure and interpretation first reported in 1996, is a sophisticated and noninvasive tool for detection of ANS regulation of the heart. ${ }^{9}$ Besides being a noninvasive study procedure, an important advantage of frequency-domain analysis of HRV is that it utilizes spontaneous fluctuations in heart rate (HR) to estimate tonic ANS functions. Investigations concerning resting HRV in PTSD revealed inconsistent results. For example, some studies found reduced resting HRV in PTSD patients compared to controls $s^{10-13}$ and a significant correlation between one of spectral HRV components and PTSD symptoms severity. ${ }^{14}$ However, other studies reported no differences in resting HRV. ${ }^{15,16}$ Moreover, a study of traumatized individuals reported greater high-frequency HRV in men with PTSD compared with men without PTSD. ${ }^{17}$ The conflicting results might be due to heterogeneity in relatively small samples, confounds from medication, physical health, habitual physical activity, smoking, psychiatric comorbidities, and reporting of different HRV measures. For example, PTSD is often associated with an increased risk of developing secondary comorbid disorders, such as depression. ${ }^{18}$ Thus, factors concomitant with depression might influence the observed relationship between PTSD and HRV. We focus on PTSD patients without depression, to avoid overestimation of the association between PTSD and HRV. Moreover, evidence indicated that Asian ethnicity served as significant predictors of healthy HRV as compared to European-American in a nonmedical sample. ${ }^{19}$ Taking ethnicity into account, studying in a genetically homogeneous population is necessary to determine the direct relation between PTSD and cardiac autonomic dysfunction. To address these concerns as mentioned above, we analyzed with larger sample sizes and better methodology. Taken together, the following hypotheses were tested:

i. Drug-naïve patients with PTSD will have decreased resting HF-HRV as compared with age- and sex-matched con- trols; and

ii. The severity of PTSD will be negatively correlated with resting HF-HRV.

Finally, the obtained results were evaluated in association with personality traits.

\section{METHODS}

\section{Subjects}

This study was approved by the Institutional Review Board for the Protection of Human Subjects at the Tri-Service General Hospital, a medical teaching hospital of the National Defense Medical Center in Taipei, Taiwan. We obtained written informed consent from all participants and fully explained the procedures of the study. Initial study entry criteria: age 2065. After detailed questionnaire screening, clinical examination and chart review, we excluded subjects with pregnancy, smoking, diabetes, cancer, neuropathy, any cardiovascular disease that affects HRV or engaging in regular physical training exceeding 10 hours a week. Subjects who used any medication that have been reported to affect the ANS functioning for at least two weeks before evaluation were also excluded.

Included in this study were outpatients with current PTSD (PTSD group), volunteers with past traumatic experiences but no current or past PTSD (past trauma group) and healthy controls without past traumatic experiences (healthy control group). Past trauma group and healthy control group were matched to PTSD group on age, gender, and education.

Based on the methodology in our previous studies, ${ }^{20}$ each patient was initially evaluated by an attending psychiatrist (HAC) and then interviewed by a well-trained psychologist, using the Chinese Version of the Modified Schedule of Affective Disorder and Schizophrenia-Lifetime (SADSL) ${ }^{21}$ to reach DSM-IV criteria for a primary diagnosis of PTSD. The interrater reliability kappa values were as follows: major depression, 0.79; bipolar disorder, 0.71; anxiety disorder, 0.86; schizophrenia, 0.95; and substance abuse and dependence, $0.82 .{ }^{22}$ Here, we further excluded individuals with a history of substance dependence, organic brain disease or any concomitant major psychiatric disorders. Moreover, all patients had never received medication that has impact on the ANS (e.g., antidepressants, antipsychotics, anticholinergics, anticonvulsants, or anxiolytics). Generally, there are a large number of PTSD patients with comorbid major depression in clinical settings. ${ }^{18}$ An attending psychiatrist used clinician-rated scales, i.e., 17-item version of the Hamilton Depression Rating Scale (HAM-D) for exclusion of depression. ${ }^{23}$ Only subjects with HAM-D score lower than 15 entered the study. ${ }^{24}$ We did this because we aimed to determine the resting HRV of a "pure" PTSD. Participants in past trauma group and he- 
althy control group were recruited from the community. We used the modified Chinese Version of SADSL to exclude individuals with psychiatric conditions. They were considered free of past or present major or minor mental illnesses. Participants in PTSD group and past trauma group experienced a variety of traumatic exposure histories. In order to match the groups with regard to lifetime history of trauma (total number of traumatic events), participants experiencing more than one lifetime traumatic event were excluded.

\section{Assessment of PTSD severity}

We used the Chinese version of the PTSD checklist-civilian version (PCL-C) to assess posttraumatic symptoms (e.g., intrusive memories). The PCL-C is a self-reported 17 -item standardized questionnaire. ${ }^{25}$ Participants rate using a 5-point scale, ranging from "not at all" to "extremely", how much the specific symptom was a problem to them over the past month. An overall score and subscores for re-experience, avoidance, and hyperarousal subscales are provided. The Chinese version of PCL-C has shown to have satisfactory reliability and validity. ${ }^{26}$

\section{Assessment of depression/anxiety severity}

All participants were interviewed by an attending psychiatrist (HAC) using the 17-item HAM-D, an objective scale to assess severity of depression. In addition, we used the Beck Depression Inventory (BDI), a 21-item questionnaire, to assess subjects' self-reported severity of depression. ${ }^{27}$ The results are scored by summing the responses to each of the items in order to obtain a total depression score (range, 0-63). All participants were assessed using self-report measures of anxiety, i.e., Beck Anxiety Inventory (BAI). ${ }^{28}$ The BAI contains 21 items that measure anxiety-related symptoms and was shown to have good test-retest reliability and fair concurrent validity. Subjects were asked to rate the severity of their anxiety on 4-point $\mathrm{Li}$ kert scales (0-3). They were also assessed by an attending psychiatrist (HAC) using clinician-rated scales, i.e., the Hamilton Anxiety Rating Scale (HAM-A). ${ }^{29}$ Both the BAI and the HAMA provide global indices of anxiety severity. To avoid multiple testing of the same hypothesis the analysis of the relationship between HRV parameters and global anxiety, severity was based on the BAI. The results were unchanged whether interviewer or self-reported measures of anxiety severity were used as an outcome.

\section{Personality assessment}

Personality traits were measured by the Tridimensional Personality Questionnaire (TPQ). The Chinese version of the TPQ used in the present study was a 100-item, self-administered, true-false instrument. The Cronbach's $\alpha$ of novelty seeking (NS) was 0.70 , and that of harm avoidance (HA) was
$0.87 .{ }^{30}$ Since the reward dependence $(\mathrm{RD})$ dimension had no adequate reliability among Han Chinese in Taiwan, ${ }^{30}$ only NS and HA dimensions were analyzed in our study. Each of the personality dimensions was postulated to be associated with a particular neurotransmitter system. Specifically, NS was mediated by the dopaminergic system and HA by the serotoninergic system. ${ }^{31}$

\section{Measurements of HRV}

Detailed procedures have been reported previously. ${ }^{32}$ In short, after sitting quietly for $20 \mathrm{~min}$, a lead I electrocardiogram was taken for $5 \mathrm{~min}$ while the subject lay quietly and breathed normally. An HRV analyzer (SSIC, Enjoy Research Inc., Taiwan) acquired, stored and processed electrocardiogram signals. Under a sampling rate of $512 \mathrm{~Hz}$, signals were recorded using an 8-bit analog-to-digital converter. Stationary R-R interval values were resampled and interpolated at a rate of 7.11 $\mathrm{Hz}$ to produce the continuity in a time domain. Power spectral analysis was performed using a nonparametric method of fast Fourier transformation (FFT). The direct current component was deleted and a Hamming window was used to attenuate the leakage effect. The power spectrum was then quantified into standard frequency-domain measurements defined previously, ${ }^{32}$ which consisted of variance (variance of RR-interval values), low frequency (LF: 0.04-0.15 Hz), high frequency (HF: $0.15-0.40 \mathrm{~Hz}$ ) and, and the ratio of LF to HF (LF/HF). All of the measurements were logarithmically transformed to correct skewed distribution. ${ }^{32}$ Vagal control of $\mathrm{HRV}$ is represented by HF, whereas both vagal and sympathetic control of HRV is jointly represented by LF. The LF/HF ratio is considered by some investigators to mirror sympathovagal balance or sympathetic modulations, with a larger $\mathrm{LF} / \mathrm{HF}$ ratio indicating a greater predominance of sympathetic activity over parasympathetic activity. ${ }^{9}$

\section{Statistical analyses}

SPSS (version 13.0, SPSS, Taipei, Taiwan) statistical software was used for all analyses. Discrete variables in patients and controls were compared using chi-square test. Differences between continuous variables were evaluated using Student's $\mathrm{t}$-test when normally distributed, otherwise the Mann-Whitney $\mathrm{U}$ test was used. To evaluate relations between variables with normal distribution, we used Pearson's correlation test. For non-normal distribution Spearman's correlation test was used. The associations between HRV measures and age, body mass index (BMI) and habitual physical activity were analyzed with product-moment correlations, whereas point-biserial correlations were used to assess relationships with gender. Results of the point-biserial correlations were identical to those arising from comparisons using $t$ tests. Linear regres- 
sion analyses were used to primarily assess associations of scores of PCL-C, BAI, BDI and TPQ with HRV indices. To control the confounding effect, we used multiple regression on the HRV indices, with HRV-associated factors as covariables. All results are two-tailed and a probability value $\mathrm{p}<0.05$ was considered statistically significant.

\section{RESULTS}

\section{Demographics and clinical characteristics}

A total of 256 subjects completed the experiments. Subjects were assigned to one of three groups: (a) PTSD group $(n=32)$, (b) past trauma group $(\mathrm{n}=32)$, and (c) healthy control group $(n=192)$. The groups did not differ significantly on demographic data, BMI, systolic/diastolic blood pressure and habitual physical activity (Table 1). As expected, group compari- sons among PTSD group, past trauma group and healthy control group showed significant differences in scores of PCL-C, HAM-A, BAI, and HA. Post hoc test revealed greater scores of four aforementioned scales in PTSD group than either past trauma group or healthy control group. There were no statistically significant differences in remaining psychometric variables in group comparisons.

\section{Heart rate variability parameters}

As can be seen in Table 2, LF and HF were significantly lower in PTSD group as compared to past trauma group and to healthy control group. Post-hoc testing showed variance in PTSD patients was lower than in healthy controls at a trend level. However, the groups were comparable regarding mean $\mathrm{R}-\mathrm{R}$ intervals and $\mathrm{LF} / \mathrm{HF}$ ratio.

Table 1. Sample characteristics

\begin{tabular}{|c|c|c|c|c|c|}
\hline Clinical and demographic data & PTSD & $\begin{array}{c}\text { Past } \\
\text { trauma }\end{array}$ & $\begin{array}{l}\text { Healthy } \\
\text { control }\end{array}$ & $\begin{array}{l}\text { Omnibus } \\
\text { p-value }\end{array}$ & $\begin{array}{l}\text { Significant } \\
\text { comparisons }\end{array}$ \\
\hline Number of participants & 32 & 32 & 192 & & \\
\hline Age, mean $\pm S D$, years & $35.53 \pm 13.48$ & $35.31 \pm 13.1$ & $36.76 \pm 12.86$ & 0.77 & \\
\hline Female sex $(\%)$ & $13(40.6)$ & $13(40.6)$ & $78(40.6)$ & 1.0 & \\
\hline Time since trauma, mean \pm SD, months & $54.69 \pm 19.3$ & $58.41 \pm 15.17$ & & 0.4 & \\
\hline Type of trauma & & & & 0.71 & \\
\hline Severe car accidents & 15 & 11 & & & \\
\hline $\begin{array}{l}\text { Injury or property damage due to natural } \\
\text { disaster or human-made disaster }\end{array}$ & 12 & 13 & & & \\
\hline $\begin{array}{l}\text { Serious physical injury due to non-motor } \\
\text { vehicle related accident }\end{array}$ & 4 & 6 & & & \\
\hline Witnessed someone seriously injured or killed & 1 & 2 & & & \\
\hline $\mathrm{BMI}$, mean $\pm \mathrm{SD}, \mathrm{kg} / \mathrm{m}^{2}$ & $22.77 \pm 2.76$ & $23.46 \pm 4.1$ & $22.59 \pm 3.31$ & 0.13 & \\
\hline Weekly regular exercise, hours & $0.63 \pm 1.56$ & $0.25 \pm 0.98$ & $0.92 \pm 1.79$ & 0.09 & \\
\hline $\mathrm{SBP}$, mean $\pm \mathrm{SD}, \mathrm{mm} \mathrm{Hg}$ & $120.34 \pm 14.84$ & $121.16 \pm 14.57$ & $119.17 \pm 15.13$ & 0.75 & \\
\hline $\mathrm{DBP}$, mean $\pm \mathrm{SD}, \mathrm{mm} \mathrm{Hg}$ & $72.38 \pm 9.67$ & $76.22 \pm 8.62$ & $74.52 \pm 10.61$ & 0.32 & \\
\hline PCL-C, mean \pm SD & $62.44 \pm 7.21$ & $18.59 \pm 1.41$ & $18.19 \pm 1.49$ & $<0.001$ & $\begin{array}{l}\text { PTSD vs. Pasttrauma, } \\
\text { PTSD vs. Healthy control }\end{array}$ \\
\hline HAM-A scores, mean \pm SD & $19.69 \pm 5.01$ & $3.75 \pm 2.81$ & $4.13 \pm 2.49$ & $<0.001$ & $\begin{array}{l}\text { PTSD vs. Pasttrauma, } \\
\text { PTSD vs. Healthy control }\end{array}$ \\
\hline BAI scores, mean \pm SD & $15.47 \pm 8.18$ & $5.56 \pm 2.78$ & $5.97 \pm 4.21$ & $<0.001$ & $\begin{array}{l}\text { PTSD vs. Pasttrauma, } \\
\text { PTSD vs. Healthy control }\end{array}$ \\
\hline HAM-D scores, mean \pm SD & $3.56 \pm 1.7$ & $3.23 \pm 1.7$ & $3.16 \pm 1.95$ & 0.62 & \\
\hline BDI scores, mean $\pm S D$ & $6.38 \pm 2.43$ & $5.06 \pm 2.87$ & $5.5 \pm 2.99$ & 0.18 & \\
\hline HA score, mean \pm SD & $19.13 \pm 7.21$ & $14.22 \pm 5.87$ & $15.17 \pm 7.06$ & 0.007 & $\begin{array}{l}\text { PTSD vs. Pasttrauma, } \\
\text { PTSD vs. Healthy control }\end{array}$ \\
\hline NS score, mean \pm SD & $15.84 \pm 3.38$ & $14.13 \pm 4.26$ & $14.71 \pm 3.92$ & 0.19 & \\
\hline
\end{tabular}

SD: standard deviation, BMI: body mass index (calculated as weight in kilograms divided by height in meters squared), SBP: systolic blood pressure, DBP: diastolic blood pressure, PCL-C: posttraumatic stress disorder checklist-civilian version, HAM-A: Hamilton Anxiety Rating Scale, BAI: Beck Anxiety Inventory, HAM-D: Hamilton Depression Rating Scale, BDI: Beck Depression Inventory, HA: harm avoidance, NS: novelty seeking 


\section{Factors associated with HRV}

Associations between HRV measures and those potentially confounding variables are summarized in Table 3. Men had significantly lower variance, LF, and HF than women. Older participants had reduced variance, $\mathrm{LF}$, and $\mathrm{HF}$, and greater LF/ HF ratio. Participants with higher BMI had greater LF/HF ratio. Participants who were habitually more physically active had significantly slower heart rates (longer RR interval) and greater LF and HF.

\section{Association between posttraumatic symptoms/ anxiety/depression severity and HRV}

Correlation analysis revealed that PCL-C scores were inversely associated with RR, variance, $L F$, and HF (Table 4).
BAI scores were associated with reduced variance, LF, and HF. The above-mentioned association remained after correction for gender, age, BMI and physical activity (Table 5). However, there was no significant correlation between severity of depression and all HRV parameters.

\section{Association between personality traits and HRV}

The HA score was inversely associated with variance, LF and $\mathrm{HF}$ among all participants (Table 4). Further adjustment for gender, age, BMI and physical activity did not alter the above-mentioned association in a meaningful way (Table 5). However, there was no significant correlation between the NS score and any HRV index in all participants.

Table 2. Mean R-R intervals (RR) and all measures of HRV for PTSD group, past trauma group and healthy control group

\begin{tabular}{lccccc}
\hline & PTSD & Past trauma & Healthy control & p-value & Significant comparisons \\
\hline $\begin{array}{l}\text { Number of participants } \\
\text { Heart rate variability measures }\end{array}$ & 32 & 32 & 192 & & \\
RR interval, mean \pm SD, ms & $848.16 \pm 124.95$ & $853.47 \pm 130.48$ & $893.48 \pm 144.34$ & 0.11 \\
Var, mean \pm SD & $7.05 \pm 0.84$ & $7.41 \pm 0.69$ & $7.44 \pm 0.88$ & 0.059 & PTSD vs. Pasttrauma, \\
LF, mean \pm SD & $5.34 \pm 1.17$ & $6.09 \pm 0.92$ & $5.9 \pm 1.07$ & 0.01 & PTSD vs. Healthy control \\
& & & & 0.003 & PTSD vs. Pasttrauma, \\
HF, mean \pm SD & $5.07 \pm 1.13$ & $5.9 \pm 0.92$ & $5.75 \pm 1.1$ & & PTSD vs. Healthy control \\
LF/HF, mean \pm SD & & & & 0.73 &
\end{tabular}

Var: total variance $\left[\ln \left(\mathrm{ms}^{2}\right)\right], \mathrm{LF}$ : low frequency power [ln $\left.\left(\mathrm{ms}^{2}\right)\right]$, HF: high frequency power [ln $\left.\left(\mathrm{ms} \mathrm{s}^{2}\right)\right]$, LF/HF: ratio of LF to HF [ln (ratio)], HRV: heart rate variability, PTSD: posttraumatic stress disorder, SD: standard deviation

Table 3. Factors associated with resting HRV indices among all participants

\begin{tabular}{lccccc}
\hline & RR interval & Var & LF & HF & LF/HF \\
\hline Gender(Women/men) $^{\dagger}$ & -0.095 & $-0.17^{* *}$ & $-0.21^{* * *}$ & $-0.13^{*}$ & -0.12 \\
Age $^{\ddagger}$ & 0.20 & $-0.45^{* * *}$ & $-0.46^{* * *}$ & $-0.51^{* * *}$ & $0.13^{*}$ \\
BMI $^{\ddagger}$ & 0.03 & 0.07 & 0.1 & -0.11 & $0.3^{* * *}$ \\
Physical activity & $0.16^{* *}$ & 0.08 & $0.13^{*}$ & $0.04^{*}$ & 0.15 \\
\hline
\end{tabular}

${ }^{*} \mathrm{p}<0.05,{ }^{* *} \mathrm{p}<0.01,{ }^{* * *} \mathrm{p}<0.001,{ }^{\dagger}$ point-biserial correlations; first category in parenthesis is the reference group, ${ }^{\ddagger}$ product-moment correlations. BMI: body mass index, Var: total variance $\left[\ln \left(\mathrm{ms}^{2}\right)\right]$, LF: low frequency power $\left[\ln \left(\mathrm{ms}^{2}\right)\right]$, HF: high frequency power [ln $\left.\left(\mathrm{ms} \mathrm{s}^{2}\right)\right], \mathrm{LF} / \mathrm{HF}: \mathrm{ratio}$ of LF to HF [ln (ratio)], HRV: heart rate variability

Table 4. The correlations between HRV indices and scores of PCL-C, BAI, BDI, HA and NS

\begin{tabular}{|c|c|c|c|c|c|}
\hline & PCL-C & BAI & BDI & $\mathrm{HA}$ & NS \\
\hline \multicolumn{6}{|l|}{ Heart rate variability measures } \\
\hline $\mathrm{RR}$ interval, mean $\pm \mathrm{SD}, \mathrm{ms}$ & $-0.19^{* *}$ & -0.002 & -0.03 & 0.05 & -0.04 \\
\hline Var, mean \pm SD & $-0.41^{* * *}$ & $-0.15^{*}$ & 0.04 & $-0.15^{*}$ & -0.01 \\
\hline $\mathrm{LF}$, mean $\pm \mathrm{SD}$ & $-0.39 * * *$ & $-0.19^{* *}$ & 0.05 & $-0.18^{* *}$ & -0.01 \\
\hline $\mathrm{HF}$, mean $\pm \mathrm{SD}$ & $-0.45^{* * *}$ & $-0.18^{* *}$ & -0.02 & $-0.21^{* *}$ & -0.02 \\
\hline $\mathrm{LF} / \mathrm{HF}$, mean $\pm \mathrm{SD}$ & 0.12 & 0.01 & 0.08 & 0.03 & 0.03 \\
\hline
\end{tabular}

${ }^{*} \mathrm{p}<0.05,{ }^{* *} \mathrm{p}<0.01,{ }^{* * *} \mathrm{p}<0.001$. Var: total variance $\left[\ln \left(\mathrm{ms}^{2}\right)\right], \mathrm{LF}$ : low frequency power $\left[\ln \left(\mathrm{ms}^{2}\right)\right]$, HF: high frequency power $\left[\ln \left(\mathrm{ms} \mathrm{s}^{2}\right)\right], \mathrm{LF} / \mathrm{HF}$ : ratio of LF to HF [ln (ratio)], PCL-C: posttraumatic stress disorder checklist-civilian version, BAI: Beck Anxiety Inventory, BDI: Beck Depression Inventory, HA: harm avoidance, NA: novelty seeking, HRV: heart rate variability 


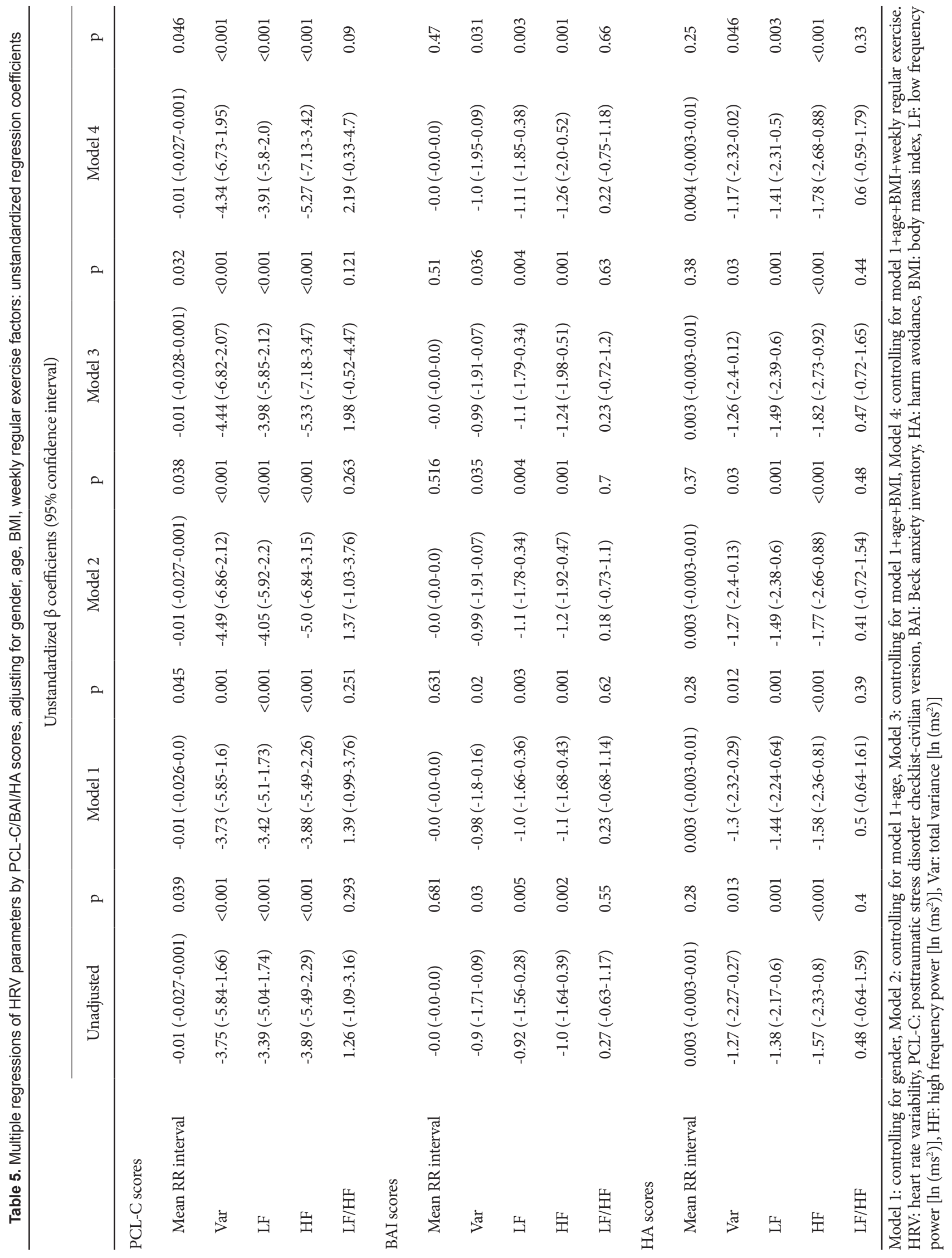




\section{DISCUSSION}

To our knowledge, this is the first adequately powered study that examines the difference of resting HRV between drugnaïve PTSD patients and controls in Han Chinese population by using frequency-domain analysis of HRV. The main results of our study may be summed as follows.

As expected, drug-naïve patients with PTSD had lower HF-HRV than either past trauma subjects or healthy controls. This result is consistent with some investigations ${ }^{11,12,33}$ and can be interpreted as evidence that the basal autonomic state of PTSD is characterized by decreased parasympathetic tone. However, our result differed from other investigations of resting HF-HRV among PTSD individuals. For example, previous investigations found no differences in baseline HFHRV when comparing PTSD patients and trauma-exposed controls. ${ }^{15,34}$ Moreover, a recent study reported that women with PTSD did not exhibit lower HF-HRV relative to agematched controls during a resting baseline period. ${ }^{16}$ We believe that our main result is reliable based on the following strengths of the present study. Our study has a larger sample size of participants than previous studies. We have excluded subjects with psychiatric and physical co-morbidities that could potentially confound the association between PTSD and cardiac autonomic functions. Three groups of subjects were interviewed with the modified Chinese Version of SADSL ${ }^{21}$ to rule out any psychiatric comorbidity or psychiatric disorder. Thus a false-positive result due to inclusion of depressive disorders, other anxiety disorders or substance use disorder in PT$\mathrm{SD}$ group is presently unlikely. We have also controlled other confounding factors that may suppress or magnify the true effects of PTSD on HRV, including medication, age, smoking, BMI and physical activity levels. ${ }^{6}$ Importantly, all our participants do not smoke or have a history of smoking. Current smoking clearly depresses HRV and, even among those who have recently quit, HRV remains lower compared with that of normal nonsmokers. ${ }^{35}$ Therefore, smoking must be taken into account in any study of the effect of PTSD on HRV because a substantial proportion of PTSD patients smoke or have a history of smoking. ${ }^{36}$ Moreover, since ethnic stratification among study samples may lead to resetting population HRV patterns, ${ }^{19,37}$ it might produce a false-positive or falsenegative result by chance rather than reveal a direct relation. However, all our subjects were unrelated Han Chinese subjects, matched for age and sex, and drawn from a population pool in Taiwan that is known to be genetically homogeneous. ${ }^{20}$ All of the biological grandparents of our recruited subjects were of Han Chinese ancestry. Therefore, it is less likely that ethnic stratification bias produced a false-positive result in our study.
Patients with more severe PTSD symptoms tend to have lower HF-HRV than those with less severe PTSD symptoms. This finding further complements our first main result regarding lower HF-HRV in PTSD. The following theoretical perspectives support our findings. Firstly, the amygdala, a limbic structure involved in emotional processing, is critical for fear conditioning and plays an important role in the pathophysiology of PTSD..$^{38}$ Neuroimaging data demonstrate that PTSD patients appear to have greater amygdala activation relative to comparison subjects. ${ }^{39}$ Neurophysiologically, the amygdale projects to the nuclei of the vagus in the brain stem, ${ }^{40}$ and may translate the psychological experiences associated with fear and trauma into the vagal responses. Secondly, Polyvagal theory proposed by Porges highlights diminished vagal tone as an important final common pathway leading to the adverse effects of acute stress reaction. ${ }^{5,15}$ Vagal influences to the heart serve to dampen the sympathetic reactions to stress and to promote calm behavioral states and self-regulation. ${ }^{5}$ Consistent with this protective function of vagal tone, a reduction in resting HF-HRV has been associated with vulnerability to the effect of stress. For example, research on PTSD suggests that early life episodes of diminished vagal tone may predict poor stress resilience in adults. ${ }^{41}$ An increase in tonic levels of HF-HRV has been observed in patients with PTSD following successful treatment. ${ }^{42}$ Altogether, our results corroborate previous work ${ }^{11-1,33}$ suggesting that reduction in CVC is a psychophysiological marker of PTSD.

Elevated resting heart rate (HR) has frequently been found in PTSD. ${ }^{12,33,42}$ Our study failed to find a HR difference. However, this is in line with other research ${ }^{15,16}$ which did not find a HR difference between PTSD and control participants. Given the large contribution of the PNS to resting HR, findings for resting HF-HRV in any single study will theoretically parallel the findings for resting HR. For example, Sahar et al. ${ }^{15}$ reported that neither resting HR nor baseline RSA (which is reflective of CVC) was different between PTSD patients and controls. However, they only studied male patients and had a small sample size. The larger sample size and drug-naïve patient population in this study made our result more reliable. Nonetheless, a meta-analysis indicated that PTSD patients had higher resting HR relative to controls. ${ }^{43}$ It would be premature to make a conclusion regarding resting HR in PTSD based up the preliminary result reported herein. In order to conclude PTSD-related alterations in resting HR, larger samples and/or meta-analyses are needed.

Of note, we observed that PTSD patients had lower LFHRV than both past trauma controls and healthy controls. Most studies reported increased sympathetic activity in PTSD. ${ }^{2,11}$ For example, Cohen et al. ${ }^{11}$ reported that the PTSD patients had increased LF-HRV compared to the controls. 
Despite our expectation that PTSD patients would be associated with a decrease in parasympathetic activity (HF-HRV) and a theoretically reciprocal increase in sympathetic activity (LF-HRV), the data shown herein indicated opposite association for sympathetic branch: lower level of LF-HRV in PTSD patients. This unexpected finding warrants comment. The traditional interpretations of the HRV measures used in our study are that HF-HRV estimate vagal tone, while LF-HRV reflects both vagal and sympathetic influences. ${ }^{9,32}$ Although some studies attribute LF fluctuations (at least in part) to sympathetic modulation, ${ }^{9}$ recent studies found no significant correlation between LF range and cardiac norepinephrine spillover. ${ }^{44}$ Thus, the LF spectral activity is likely a poor marker of sympathetic outflow ${ }^{45}$ and conclusions regarding changes in sympathetic branch could not be drawn from the short-term HRV linear analysis. Furthermore, it has also been reported that when LF-HRV is assessed in the supine position, administration of atropine, a potent inhibitor of parasympathetic muscarinic receptors, eliminates most of the LF region of the power spectrum. ${ }^{46}$ This does not occur when LF-HRV is assessed in the sitting position, and suggests that resting LFHRV in our study may primarily reflect vagal influences. ${ }^{47}$ Correlations with other clearly sympathetic measures, such as skin conductance, skin temperature, or muscle sympathetic nerve activity, should be investigated in the future.

Finally, we found a negative correlation between CVC (HFHRV) and the HA score. HA corresponds to an inhibitory response to signals of aversive stimuli that leads to avoidance of punishment, and it is theoretically associated with serotonergic activity. ${ }^{31,48}$ For example, evidence indicated that the HA score was positively associated with serotonin (5-HT) receptor sensitivity ${ }^{48}$ and negatively correlated with serotonin transporter (5-HTT) availability. ${ }^{49}$ Genetic studies further proved the relationship between the HA score and the serotonin transporter gene-linked polymorphic region. ${ }^{50}$ Based on previous findings high HA score seems to reflect lowered 5-HT function. Then, a link between lowered 5-HT function and reduced HRV was established by recent evidence indicating that 5-HT depletion or 5-HT receptor blocking attenuated baroreflex gain. ${ }^{51,52}$ The arterial baroreflex is the main mediator of HRV; ${ }^{53}$ therefore, lowered 5-HT function leads to diminished baroreflex function which in turn leads to reduced HRV. Altogether, our work and other results cited above relate the degree of reduction in HRV to the degree of HA which may be reflective of central serotonergic activity. To date, the mechanism involved in bringing about alterations of neurocardiac regulation in PTSD is yet to be established. It is well known that PTSD is associated with altered 5-HT neurotransmission, as evidenced by 5-HT2A receptor polymorphism in increasing genetic risk for $\mathrm{PTSD}^{54}$ and de- creased serum concentrations of 5-HT and decreased density of platelet 5-HT uptake sites in PTSD patients. ${ }^{55,56}$ A plausible scenario may be that decreased 5-HT transmission in PTSD may eventually have a role in causing cardiac autonomic dysregulation. Consistent with such a possibility, Cohen et al. ${ }^{42}$ reported that the HRV parameters indicating cardiac autonomic dysregulation, which characterizes PTSD patients at rest, were normalized in responding patients by use of fluoxetine, a selective serotonin re-uptake inhibitor (SSRI).

As suggested above, our findings highlight decreased CVC in PTSD patients and should serve to remind clinicians to pay attention to their increased risk of cardiovascular diseases. For example, an autonomic function examination such as HRV analysis can be done to provide a rapid screening of systemic autonomic disturbance. In the present study, we assumed that decreased CVC in PTSD may be associated with impairment of serotonergic activity. SSRIs have been recommended as first-line agents to treat PTSD indeed. ${ }^{57}$ Nevertheless, a recent review emphasizes high drop-out rates in the SSRI trials, and the negative trials in subjects with combatrelated PTSD. ${ }^{58}$ There is a paucity of data on changes in cardiac autonomic function of PTSD patients treated with SSRI. ${ }^{42}$ Accordingly, the alternative treatment to restore the autonomic function may be considered for PTSD patients. For instance, preliminary results suggested that HRV biofeedback $^{59}$ and autogenic training ${ }^{8}$ significantly increased HRV and/or CVC while reducing symptoms of PTSD. Larger randomized control trials to validate these study results are warranted.

Several limitations should be considered in the present study. 1) The study utilized cross-sectional data only, and causality therefore cannot be inferred. 2) Interpretation of LFHRV as sympathetic activity should be made with caution, as the level of modulation of LF-HRV by the sympathetic branch is still debated. 3) Our study failed to provide the information concerning the menstrual cycle of our female participants; however, HRV was shown to fluctuate during different phases of female menstrual cycle. ${ }^{60}$ 4) All our participants were physically healthy, so our results may not generalize to other patient populations. Because noise and arrhythmias can greatly affect the accuracy of HRV estimation, the use of HRV techniques is hampered in certain patients (e.g., uncooperative, mentally ill or medically ill patients). Frequency domain measures of HRV are also difficult to validate and standardize. $^{61}$ All these factors weaken HRV analysis as scientific measure for clinical application.

\section{Acknowledgments}

This study was supported by Tri-Service General Hospital Grant TSGHC98-91 and TSGH-C102-123 (H.A.C). The authors thank psychologist Tsuey-Yen Yeh for her assistance in the interviewing. 


\section{REFERENCES}

1. Kessler RC. Posttraumatic stress disorder: the burden to the individual and to society. J Clin Psychiatry 2000;61(Suppl 5):4-12.

2. Peri T, Ben-Shakhar G, Orr SP, Shalev AY. Psychophysiologic assessment of aversive conditioning in posttraumatic stress disorder. Biol Psychiatry 2000;47:512-519.

3. Shalev AY, Peri T, Gelpin E, Orr SP, Pitman RK. Psychophysiologic assessment of mental imagery of stressful events in Israeli civilian posttraumatic stress disorder patients. Compr Psychiatry 1997;38:269-273.

4. Murburg MM, McFall ME, Lewis N, Veith RC. Plasma norepinephrine kinetics in patients with posttraumatic stress disorder. Biol Psychiatry 1995;38:819-825.

5. Porges SW. The polyvagal theory: phylogenetic substrates of a social nervous system. Int J Psychophysiol 2001;42:123-146.

6. Rottenberg J. Cardiac vagal control in depression: a critical analysis. Biol Psychol 2007;74:200-211.

7. Shaikh al arab A, Guedon-Moreau L, Ducrocq F, Molenda S, Duhem S, Salleron J, et al. Temporal analysis of heart rate variability as a predictor of post traumatic stress disorder in road traffic accidents survivors. J Psychiatr Res 2012;46:790-796.

8. Mitani S, Fujita M, Sakamoto S, Shirakawa T. Effect of autogenic training on cardiac autonomic nervous activity in high-risk fire service workers for posttraumatic stress disorder. J Psychosom Res 2006;60:439444 .

9. Heart rate variability: standards of measurement, physiological interpretation and clinical use. Task Force of the European Society of Cardiology and the North American Society of Pacing and Electrophysiology. Circulation 1996;93:1043-1065.

10. Cohen H, Kotler M, Matar MA, Kaplan Z, Loewenthal U, Miodownik H, et al. Analysis of heart rate variability in posttraumatic stress disorder patients in response to a trauma-related reminder. Biol Psychiatry 1998; 44:1054-1059.

11. Cohen H, Kotler M, Matar MA, Kaplan Z, Miodownik H, Cassuto Y. Power spectral analysis of heart rate variability in posttraumatic stress disorder patients. Biol Psychiatry 1997;41:627-629.

12. Cohen H, Benjamin J, Geva AB, Matar MA, Kaplan Z, Kotler M. Autonomic dysregulation in panic disorder and in post-traumatic stress disorder: application of power spectrum analysis of heart rate variability at rest and in response to recollection of trauma or panic attacks. Psychiatry Res 2000;96:1-13.

13. Hauschildt M, Peters MJ, Moritz S, Jelinek L. Heart rate variability in response to affective scenes in posttraumatic stress disorder. Biol Psychol 2011;88:215-222.

14. Song BA, Yoo SY, Kang HY, Byeon SH, Shin SH, Hwang EJ, et al. Posttraumatic stress disorder, depression, and heart-rate variability among North Korean defectors. Psychiatry Investig 2011;8:297-304.

15. Sahar T, Shalev AY, Porges SW. Vagal modulation of responses to mental challenge in posttraumatic stress disorder. Biol Psychiatry 2001;49: 637-643.

16. Keary TA, Hughes JW, Palmieri PA. Women with posttraumatic stress disorder have larger decreases in heart rate variability during stress tasks. Int J Psychophysiol 2009;73:257-264.

17. Kamkwalala A, Norrholm SD, Poole JM, Brown A, Donley S, Duncan E, et al. Dark-enhanced startle responses and heart rate variability in a traumatized civilian sample: putative sex-specific correlates of posttraumatic stress disorder. Psychosom Med 2012;74:153-159.

18. Ballenger JC, Davidson JR, Lecrubier Y, Nutt DJ, Foa EB, Kessler RC, et al. Consensus statement on posttraumatic stress disorder from the International Consensus Group on Depression and Anxiety. J Clin Psychiatry 2000;61(Suppl 5):60-66.

19. Martin LA, Doster JA, Critelli JW, Lambert PL, Purdum M, Powers C, et al. Ethnicity and Type $\mathrm{D}$ personality as predictors of heart rate variability. Int J Psychophysiol 2010;76:118-121.

20. Chang CC, Lu RB, Chen CL, Chu CM, Chang HA, Huang CC, et al.
Lack of association between the norepinephrine transporter gene and major depression in a Han Chinese population. J Psychiatry Neurosci 2007;32:121-128.

21. Endicott J, Spitzer RL. A diagnostic interview: the schedule for affective disorders and schizophrenia. Arch Gen Psychiatry 1978;35:837-844.

22. Huang SY, Lin WW, Ko HC, Lee JF, Wang TJ, Chou YH, et al. Possible interaction of alcohol dehydrogenase and aldehyde dehydrogenase genes with the dopamine $\mathrm{D} 2$ receptor gene in anxiety-depressive alcohol dependence. Alcohol Clin Exp Res 2004;28:374-384.

23. Hamilton M. A rating scale for depression. J Neurol Neurosurg Psychiatry 1960;23:56-62.

24. Frank E, Prien RF, Jarrett RB, Keller MB, Kupfer DJ, Lavori PW, et al. Conceptualization and rationale for consensus definitions of terms in major depressive disorder. Remission, recovery, relapse, and recurrence. Arch Gen Psychiatry 1991;48:851-855.

25. Blanchard EB, Jones-Alexander J, Buckley TC, Forneris CA. Psychometric properties of the PTSD Checklist (PCL). Behav Res Ther 1996; 34:669-673.

26. Wu KK, Chan SK, Yiu VF. Psychometric properties and confirmatory factor analysis of the posttraumatic stress disorder checklist for Chinese survivors of road traffic accidents. Hong Kong J Psychiatry 2008; 18:144-151.

27. Beck AT, Ward CH, Mendelson M, Mock J, Erbaugh J. An inventory for measuring depression. Arch Gen Psychiatry 1961;4:561-571.

28. Beck AT, Steer RA. Beck Anxiety Inventory Manual. San Antonio: The Psychological Corporation Harcourt; 1990.

29. Hamilton M. The assessment of anxiety states by rating. Br J Med Psychol 1959;32:50-55.

30. Chen WJ, Chen HM, Chen CC, Yu WY, Cheng AT. Cloninger's Tridimensional Personality Questionnaire: psychometric properties and construct validity in Taiwanese adults. Compr Psychiatry 2002;43:158166.

31. Cloninger CR. Neurogenetic adaptive mechanisms in alcoholism. Science 1987;236:410-416.

32. Kuo TB, Lin T, Yang CC, Li CL, Chen CF, Chou P. Effect of aging on gender differences in neural control of heart rate. Am J Physiol 1999; 277:H2233-H2239.

33. Blechert J, Michael T, Grossman P, Lajtman M, Wilhelm FH. Autonomic and respiratory characteristics of posttraumatic stress disorder and panic disorder. Psychosom Med 2007;69:935-943.

34. Woodward SH, Kaloupek DG, Schaer M, Martinez C, Eliez S. Right anterior cingulate cortical volume covaries with respiratory sinus arrhythmia magnitude in combat veterans. J Rehabil Res Dev 2008;45:451-463.

35. Stein PK, Rottman JN, Kleiger RE. Effect of $21 \mathrm{mg}$ transdermal nicotine patches and smoking cessation on heart rate variability. Am J Cardiol 1996;77:701-705.

36. Beckham JC, Gehrman PR, McClernon FJ, Collie CF, Feldman ME. Cigarette smoking, ambulatory cardiovascular monitoring, and mood in Vietnam veterans with and without chronic posttraumatic stress disorder. Addict Behav 2004;29:1579-1593.

37. Li Z, Snieder H, Su S, Ding X, Thayer JF, Treiber FA, et al. A longitudinal study in youth of heart rate variability at rest and in response to stress. Int J Psychophysiol 2009;73:212-217.

38. Davis M, Walker DL, Lee Y. Roles of the amygdala and bed nucleus of the stria terminalis in fear and anxiety measured with the acoustic startle reflex. Possible relevance to PTSD. Ann N Y Acad Sci 1997;821:305-331.

39. Liberzon I, Sripada CS. The functional neuroanatomy of PTSD: a critical review. Prog Brain Res 2008;167:151-169.

40. Schwaber JS, Kapp BS, Higgins G. The origin and extent of direct amygdala projections to the region of the dorsal motor nucleus of the vagus and the nucleus of the solitary tract. Neurosci Lett 1980;20:15-20.

41. Bracha HS. Can premorbid episodes of diminished vagal tone be detected via histological markers in patients with PTSD? Int J Psychophysiol 2004;51:127-133.

42. Cohen H, Kotler M, Matar M, Kaplan Z. Normalization of heart rate 
variability in post-traumatic stress disorder patients following fluoxetine treatment: preliminary results. Isr Med Assoc J 2000;2:296-301.

43. Buckley TC, Kaloupek DG. A meta-analytic examination of basal cardiovascular activity in posttraumatic stress disorder. Psychosom Med 2001;63:585-594.

44. Baumert M, Lambert GW, Dawood T, Lambert EA, Esler MD, McGrane M, et al. Short-term heart rate variability and cardiac norepinephrine spillover in patients with depression and panic disorder. Am J Physiol Heart Circ Physiol 2009;297:H674-H679.

45. Moak JP, Goldstein DS, Eldadah BA, Saleem A, Holmes C, Pechnik S, et al. Supine low-frequency power of heart rate variability reflects baroreflex function, not cardiac sympathetic innervation. Heart Rhythm 2007;4:1523-1529.

46. Pomeranz B, Macaulay RJ, Caudill MA, Kutz I, Adam D, Gordon D, et al. Assessment of autonomic function in humans by heart rate spectral analysis. Am J Physiol 1985;248:H151-H153.

47. Sloan RP, McCreath H, Tracey KJ, Sidney S, Liu K, Seeman T. RR interval variability is inversely related to inflammatory markers: the CARDIA study. Mol Med 2007;13:178-184.

48. Peirson AR, Heuchert JW, Thomala L, Berk M, Plein H, Cloninger CR. Relationship between serotonin and the temperament and character inventory. Psychiatry Res 1999;89:29-37.

49. Wu IT, Lee IH, Yeh TL, Chen KC, Chen PS, Yao WJ, et al. The association between the harm avoidance subscale of the Tridimensional Personality Questionnaire and serotonin transporter availability in the brainstem of male volunteers. Psychiatry Res 2010;181:241-244.

50. Lesch KP, Bengel D, Heils A, Sabol SZ, Greenberg BD, Petri S, et al. Association of anxiety-related traits with a polymorphism in the serotonin transporter gene regulatory region. Science 1996;274:1527-1531.

51. Damaso EL, Bonagamba LG, Kellett DO, Jordan D, Ramage AG, Mach- ado BH. Involvement of central 5-HT7 receptors in modulation of cardiovascular reflexes in awake rats. Brain Res 2007;1144:82-90.

52. Kellett DO, Ramage AG, Jordan D. Central 5-HT7 receptors are critical for reflex activation of cardiac vagal drive in anaesthetized rats. J Physiol 2005;563:319-331.

53. Frederiks J, Swenne CA, TenVoorde BJ, Honzikova N, Levert JV, Maan $\mathrm{AC}$, et al. The importance of high-frequency paced breathing in spectral baroreflex sensitivity assessment. J Hypertens 2000;18:1635-1644.

54. Lee HJ, Kwak SK, Paik JW, Kang RH, Lee MS. Association between serotonin $2 \mathrm{~A}$ receptor gene polymorphism and posttraumatic stress disorder. Psychiatry Investig 2007;4:104-108.

55. Vermetten E, Bremner JD. Circuits and systems in stress. II. Applications to neurobiology and treatment in posttraumatic stress disorder. Depress Anxiety 2002;16:14-38.

56. Ressler KJ, Nemeroff CB. Role of serotonergic and noradrenergic systems in the pathophysiology of depression and anxiety disorders. Depress Anxiety 2000;12(Suppl 1):2-19.

57. Song JM, Chae JH. Update on current treatment options for posttraumatic stress disorder. Psychiatry Investig 2005;2:31-43.

58. Stein DJ, Ipser J, McAnda N. Pharmacotherapy of posttraumatic stress disorder: a review of meta-analyses and treatment guidelines. CNS Spectr 2009;14:25-31.

59. Tan G, Dao TK, Farmer L, Sutherland RJ, Gevirtz R. Heart rate variability (HRV) and posttraumatic stress disorder (PTSD): a pilot study. Appl Psychophysiol Biofeedback 2011;36:27-35.

60. Sato N, Miyake S, Akatsu J, Kumashiro M. Power spectral analysis of heart rate variability in healthy young women during the normal menstrual cycle. Psychosom Med 1995;57:331-335.

61. Kleiger RE, Stein PK, Bigger JT Jr. Heart rate variability: measurement and clinical utility. Ann Noninvasive Electrocardiol 2005;10:88-101. 